\title{
Cesación de tabaquismo en embarazo
}

\author{
JAIME CERDA L.*
}

\section{Smoking cessation in pregnancy}

In Chile, women of childbearing age and pregnant women have a high prevalence of smoking. Tobacco use during pregnancy has antenatal effects (spontaneous abortion, stillbirth) and it is a risk factor for infant morbidity and mortality. All women of childbearing age should be encouraged to quit smoking, and women who are already pregnant should be encouraged to stop smoking continuously throughout the pregnancy, from the earliest possible moment to the postpartum period. We present a set of background information and recommendations for smoking cessation in pregnant women, based on international guidelines on this topic.

Key words: Smoking cessation; pregnant women; tobacco use disorder.

\section{Resumen}

En Chile, las mujeres en edad fértil y las embarazadas presentan una alta prevalencia de tabaquismo. El consumo de tabaco durante el embarazo tiene efectos prenatales (por ejemplo, aborto espontáneo, mortinatalidad) y es un factor de riesgo de morbilidad y mortalidad infantil. Todas las mujeres en edad fértil deben ser alentadas a dejar de fumar, y las mujeres que ya están embarazadas deben ser alentadas a dejar de fumar continuamente durante todo el embarazo, desde el momento más precoz posible hasta el periodo posterior al parto. Se presenta un conjunto de antecedentes y recomendaciones para la cesación de tabaquismo en embarazadas, basadas en guías internacionales sobre este tema.

Palabras clave: Dejar de fumar; mujeres embarazadas; tabaquismo.

\section{Antecedentes}

En Chile, las mujeres en edad fértil presentan una prevalencia de fumador actual de $42,6 \%(15-$ 24 años) y 44,3\% (25-44 años). En promedio, el número de cigarrillos diarios fumados en mujeres en edad fértil es 6,0 (15-24 años) y 9,7 (25-44 años) $)^{1}$.

La prevalencia de consumo de tabaco en Chile durante el embarazo ha sido estimada en diversos estudios, destacando el de Mallol y cols (2007) $)^{2}$, consistente en una evaluación de 400 puérperas provenientes de las comunas de San Bernardo y El Bosque, entrevistadas por un pediatra. Un 28\% de las puérperas reportó haber fumado durante su último embarazo y $68 \%$ reportó consumo de tabaco previo al embarazo (45,1\% diariamente y $22,9 \%$ ocasionalmente). Por su parte, los estudios de Telgie (2007) $)^{3}$ y Pérez-Franco y Raffo $(2015)^{4}$ reportaron prevalencias de consumo de tabaco prenatal de $31,5 \%$ y $36,4 \%$, respectivamente.

El consumo de tabaco durante el embarazo tiene efectos prenatales (por ejemplo, aborto espontáneo, mortinatalidad) y es un factor de riesgo de morbilidad y mortalidad infantil. Destaca su rol en la génesis de prematurez, bajo peso de nacimiento, complicaciones respiratorias y síndrome de muerte súbita del lactante ${ }^{5}$. Dejar de fumar lo antes posible durante el embarazo puede reducir los riesgos antes mencionados.

Cuando las mujeres embarazadas dejan de fumar, tanto la madre como el hijo se benefician. Todas las mujeres en edad fértil deben ser alentadas a dejar de fumar, y las mujeres que ya están embarazadas deben ser alentadas a dejar de fumar continuamente durante todo el embarazo (desde

* Dpto. Salud Pública, Facultad de Medicina, Pontificia Universidad Católica de Chile. 
el momento más precoz posible hasta el período posterior al parto). Prevenir el daño a su bebé por nacer es un fuerte motivador para que las mujeres embarazadas dejen de fumar ${ }^{6}$.

A pesar de la ocurrencia de recaídas, se debe ofrecer apoyo continuo a la mujer para permanecer libre de humo después del nacimiento de su hijo. La nicotina pasa libremente hacia y desde la leche materna, dependiendo de la concentración de nicotina en la sangre materna (que se ve afectada por el consumo de cigarrillos, la frecuencia de la lactancia materna y el tiempo entre fumar y amamantar). Es importante enfatizar la importancia de seguir amamantando, independientemente del hábito tabáquico. También se sabe que el humo de tabaco de segunda mano tiene efectos perjudiciales para la salud de los niños pequeños ${ }^{6}$.

\section{Recomendaciones $^{7,8}$}

[(Basadas en la guía confeccionada por NICE $(2010)^{7}$ y en la European Tobacco Treatment Guideline, TOB.g $\left.(2017)^{8}\right]$.

1. Identifique a la embarazada o madre que fuma, lo más precozmente posible. Para algunas mujeres es difícil decir que fuman debido a que existe un estigma social asociado a fumar durante el embarazo. Todos los integrantes del equipo de salud encargado de los cuidados de la embarazada, la madre y el bebé deberían intentar identificar a la embarazada o madre que fuma. Ello incluye también otros profesionales (por ejemplo, odontólogos, parvularias, asistentes sociales). En todos los encuentros de la embarazada o madre con el sistema de salud se le debería preguntar por su consumo de tabaco. Pregunte a la embarazada o madre cuántos cigarrillos fuma, qué tan frecuente lo hace y averigüe cualquier inquietud que ella, su pareja o su familia pudiesen tener acerca de dejar de fumar. Si la mujer declara no fumar, dé un refuerzo positivo.

2. Realice una consejería breve que incluya información sobre los riesgos de fumar durante el embarazo para el feto en gestación y sobre el peligro de exponerse a humo de segunda mano tanto para el bebé como para la madre. Explique los beneficios que tiene el dejar de fumar para la salud de la mujer y de su bebé. Aconseje de manera directa y firme dejar de fumar, y no simplemente reducir el consumo. No existe un nivel seguro de tabaquismo en el embarazo.

3. Entregue a la mujer apoyo para la cesación de consumo de tabaco durante el embarazo y posterior a éste. Sea sensible a las circunstancias difíciles en que se encuentran muchas embarazadas que fuman. Considere también otros factores tales como la edad de la mujer y su nivel de adicción, entre otros.

4. Uso de terapia farmacológica.

- La evidencia disponible es insuficiente para evaluar el balance de beneficios y daños de la terapia farmacológica en embarazadas.

- Ni la vareniclina ni el bupropión deben ofrecerse a mujeres embarazadas o que están dando de mamar.

- Si una embarazada expresa un claro deseo de recibir terapia de reemplazo de nicotina (TRN), se sugiere (i) discutir con ella los riesgos y beneficios asociados, (ii) utilizarla sólo si falla la cesación con medidas no farmacológicas y (iii) utilizar el criterio profesional al decidir si ofrecer la prescripción de TRN, tomando en cuenta el nivel de adicción de la embarazada y la presencia de comorbilidades.

- Si bien no existe consenso en el esquema terapéutico a usar en embarazadas, expertos internacionales sugieren lo siguiente:

Partir con chicles de nicotina de $2 \mathrm{mg}$. Si esto no funciona considerar el uso de parches de nicotina de $24 \mathrm{~h}$ de duración que se colocan en la mañana y se retiran en la noche. Si la paciente fuma menos de 10 cigarrillos al día usar 1 parche al día de 7 o $14 \mathrm{mg}$. Si fuma 10 o más cigarrillos al día partir con 1 parche al día de $14 \mathrm{mg}$. Evaluar cada 2 semanas y considerar la disminución progresiva de los parches de 14 a $7 \mathrm{mg}$. Usar por un máximo de 2 meses. Notar que solo se justifica mantener los parches si la paciente se mantiene sin fumar.

Basado en esquema terapéutico usado en The Mayo Clinic Dependence Center, USA y Division of Primary Care, University of Nottingham, England.

5. Si la pareja u otro integrante en el hogar fuma, sugiérale dejar de fumar. Si nadie fuma en el hogar, de un refuerzo positivo. Recomiende no fumar alrededor de la mujer embarazada, madre o bebé. Ello incluye no fumar en la casa o en el automóvil.

\section{Bibliografía}

1.- Ministerio de Salud - Chile. Encuesta Nacional de Salud (Chile 2010). Disponible en: http://web.minsal.cl/portal/ url/item/bcb03d7bc28b64dfe040010165012d23.pdf (consultado el 19/09/2017).

2.- MALLOL J, BRANDENBURG D, MADRID R, 
SEMPERTEGUI F, RAMÍREZ L, JORQUERA D Prevalencia de tabaquismo durante el embarazo en mujeres chilenas de bajo nivel socioeconómico. Rev Chil Enferm Respir 2007; 23: 17-22.

3.- TELGIE R. Prevalencia de fumar en las gestantes controladas en los consultorios municipalizados de la comuna de Quinta Normal (Región Metropolitana, Chile). Cuad Med Soc (Chile) 2007; 47: 253-7.

4.- PÉREZ-FRANCO J, RAFFO S. Uso de sustancias psicoactivas en el embarazo y conocimiento de los efectos sobre el feto en puérperas recientes. Rev Chil Salud Pública 2015; 19: 37-46.

5.- DIETZ P, ENGLAND L, SHAPIRO-MENDOZA C, TONG V, FARR S, CALLAGHAN W. Infant morbidity and mortality attributable to prenatal smoking in the U.S. Am J Prev Med 2010; 39: 45-52.
6.- Ministerio de Salud - Gobierno de Nueva Zelandia. Antecedentes y recomendaciones de las directrices de Nueva Zelandia para ayudar a las personas a dejar de fumar. Disponible en: http://www.health.govt.nz/publication/ new-zealand-guidelines-helping-people-stop-smoking (consultado el 19/09/2017).

7.- National Institute for Healthcare Excellence (NICE). Smoking: stopping in pregnancy and after childbirth. Public Health Guideline [PH26] (2010). Disponible en: https://www.nice.org.uk/guidance/ph26 (consultado el 19/09/2017).

8.- European Tobacco Treatment Guideline. Tobacco Cessation Guidelines for High-Risk Populations (TOB.g). Disponible en: http://tob-g.eu/wp-content/uploads/ TOB-G-BOOK-DIGITAL-VERSION.pdf (consultado el 19/09/2017).

Correspondencia a:

Dr. Jaime Cerda L.

Email:jcerda@uc.cl 